\title{
C.487C > T mutation in PAX4 gene causes MODY9 - a case report and literature review
}

\author{
Di Zhang ${ }^{1}$, Wenli Yang ${ }^{2}$, Li Ren $^{3}$, and yanmei sang ${ }^{4}$ \\ ${ }^{1}$ Capital Institute of Pediatrics \\ ${ }^{2}$ Beijing Children's Hospital \\ ${ }^{3}$ Beijing Children's Hospital Affiliated to Capital Medical University \\ ${ }^{4}$ Beijing Children's Hospital
}

November 18, 2020

\begin{abstract}
Maturity-onset diabetes of the young (MODY) is a group of monogenic diabetes characterized by an autosomal dominant inheritance. MODY is categorized by a large variety of clinical forms and is caused by a wide spectrum of mutations in MODY-related genes that lead to different clinical pictures and require distinct treatment
\end{abstract}

\section{C.487C $>$ T mutation in $P A X 4$ gene causes MODY9 - a case report and literature review} Di Zhang ${ }^{1}$ Wenli Yang ${ }^{2}$ Li Ren ${ }^{2}$ Yanmei Sang ${ }^{2}$

${ }^{1}$ Capital Institute Of Pediatrics

${ }^{2}$ Beijing Children's Hospital

\begin{abstract}
Objective: Maturity-onset diabetes of the young (MODY) is a group of monogenic diabetes characterized by an autosomal dominant inheritance. MODY is categorized by a large variety of clinical manifestations and is caused by a wide spectrum of mutations in MODY-related genes that lead to different clinical pictures and require distinct treatment strategies. In the current study, the clinical features and gene mutations of a Chinese child, diagnosed with MODY9, are reported for the first time with the relevant literature being adequately reviewed. Methods: The clinical data of a PAX4-MODY9 patient admitted to the Department of Endocrinology at Beijing Children's Hospital are summarized, and his genetic sequencing results are analyzed and discussed. Results: The patient was male, aged 19 months. He was admitted to the hospital due to excessive water drinking, polyuria for over half a month and wheezing for 3 days. Laboratory examination showed that the blood glucose was $21.69 \mathrm{mmol} / \mathrm{L}$, glycosylated haemoglobin was $11.1 \%$, the measured levels of insulin on the $0^{\text {th }}$ minute were $0.56 \mu \mathrm{IU} / \mathrm{ml}$, while at the same time point the C-peptide blood serum concentration was $0.09 \mathrm{ng} / \mathrm{ml}$. ICA (anti-islet cell antibody) and GADA (antiglutamate decarboxylase antibody) were negative. Blood gas analysis showed that the blood pH was 7.06, the actual and standard bicarbonate was $4.2 \mathrm{mmol} / \mathrm{L}$ and $5.8 \mathrm{mmol} / \mathrm{L}$, while the whole blood base storage was $24.2 \mathrm{mmol} / \mathrm{L}$. Urine routine tests showed that urine glucose and ketone bodies were positive, $4+$ and $3+$ respectively. The patient was treated with insulin in the meantime. The whole-exon sequencing analysis demonstrated that the child carried the heterozygous missense mutation of c.487>T in the $7^{\text {th }}$ exon region of PAX4 gene, leading to the amino acid sequence variation of p.R163W. The father of the child patient had the same heterozygous mutation, while the mother presented with a normal genotype, suggesting that the mutation was autosomal dominant. Up to now, c. $487 \mathrm{C}>\mathrm{T}$ mutation in the PAX4 gene has not been reported
\end{abstract}


in literature yet. Conclusions: MODY diagnostics by gene sequencing analysis holds great potential for precise and on time therapeutic solutions. Results show that in Chinese children, the rare heterozygous mutation c.487C $>$ T of PAX4 gene leads to the occurrence of MODY9 and though the clinical symptoms of MODY9 are different, we suggest this patient to undergo insulin therapy.

Keywords: MODY9, PAX4 gene, missense mutation, next-generation sequencing, monogenic diabetes

\section{Key Clinical Message}

A novel heterozygous mutation of the PAX4 gene $(\mathrm{c} .487 \mathrm{C}>\mathrm{T})$ leads to the occurrence of MODY9 in an 1-year-old Chinese patient.

Gene sequencing analysis plays a vital role in the diagnosis and classification of MODY.

\section{Introduction}

Maturity-onset diabetes of the young (MODY) is a group of monogenic diabetes (MD) characterized by an autosomal dominant inheritance. MD is a specific type of diabetes resulting from single-gene mutations that play a key role in the development, function, and insulin signalling pathway of pancreatic $\beta$-cells. It accounts for approximately 1 to $5 \%$ of all types of diabetes [1,2] and mainly includes MODY, neonatal diabetes, mitochondrial gene mutation diabetes, adipose atrophic diabetes and others. Besides, insulin resistance syndrome caused by a single-gene mutation presenting with diabetes as one of the main clinical manifestations also belongs to the category of monogenic diabetes. Among them, MODY accounts for approximately $1 \%$ of the total population with diabetes, and about 1 to $2 \%$ of diabetes in the Chinese population [3]. The population characteristics of MODY mainly include an early onset before the age of 25 , confirmed family history of multiple generations with diabetes together with an autosomal dominant inheritance pattern. Recent data show that MODY1 is the first-named MODY subtype, and its pathogenic gene is the hepatocellular nuclear factor (HNF), namely, 4 $\alpha$. It was first named in 1996 by the Japanese scholar Yamagouta et al. [4]. With the continuous progress of research, 14 MODY pathogenic genes have been found so far, and correspondingly 14 subtypes have been classified. They are hepatocyte nuclear factor $4 \alpha$, HNF- $4 \alpha$, MODY1, glucokinase, GCK, MODY2, hepatocyte nuclear factor $1 \alpha$ HNF-1 $\alpha$, MODY3, insulin promoter factor 1,I PF-1, MODY4, hepatocyte nuclear factor 1 $\beta$, HNF1- $\beta$, MODY5, neurogenic differentiation factor, NEUROD-1, MODY6, kruppel-like factor 11, KLF-11, MODY7, carboxyl-ester lipase, CEL, MODY8, paired-homeodomain transcription factor, PAX-4, MODY 9, insulin gene, INS, MODY10, B-lymphocyte kinase, BLK, MODY11, ATP-binding cassette, sub-family C member 8, ABCC8, MODY12, potassium inwardly-rectifying channel, subfamily J member 11, KCAJ-11, MODY13 and adaptor protein, phosphotyrosine-interacting PH domain and leucine zipper 1, APPL1, and MODY14. Among them, the most common types in the Chinese population are MODY1, MODY2, and MODY3, respectively [5]. Relevant research data show that about 16 to $45 \%$ of MODY gene loci have not been recognized so far and are currently temporarily called candidate genes of MODY-X. Possible candidate genes include paired box gene 6, PAX6, hepatocyte nuclear factor-3 $\beta$, HNF-3 $\beta$, Kappa2.2, NKX 2.2, Neurogenin3, Ngn3, and Nuclear transcription factor, NFX6.1 [6-8].

Due to the atypical clinical manifestations of MODY, pediatricians face serious difficulties in the clinical understanding of the disease. In clinical practice, MODY is often misdiagnosed as type 1 or type 2 diabetes, and therefore receive inappropriate overtreatment. Typical MODY cases are characterized by an early onset before the age of 25, pancreatic $\beta$-cell functional disorder and other features [9]. Recent data show that patients with MODY account for about 1 to $2 \%$ of all children diagnosed with diabetes. Interestingly, in these patients, the pancreatic autoantibodies are negative while the serum levels of C-peptide usually are in the normal range or slightly lower, which allows the distinction of MODY from type 1 diabetes. Normally, MODY patients are not obese and are therefore distinguished from type 2 diabetes patients. Research in this field has discovered 14 kinds of MODY subtypes so far. Therefore, for accurate treatment, prognostic evaluation and prenatal consultation, it is of great significance to precisely distinguish MODY patients from those with type 1 and 2 diabetes. 
MODY9, first reported in 2007 [10], is caused by a mutation in the Paired box gene 4 (PAX4) and is a rare type of MODY. PAX4, a member of the PAX transcription factor family, is an important regulator of early pancreatic development in the embryonic stage and plays an important role in the differentiation and development of islet $\beta$-cells [11]. Research data show that the incidence of MODY9 in populations with MODY is low. So far, only a few families have been reported with no confirmed case of MODY9 in China.

Here, we report on a case of a Chinese MODY9 patient diagnosed and treated at the Department of Endocrinology at Beijing Children hospital. We further discuss the relevant literature to improve clinicians' understanding of this disease and to propose modern approaches for precise and on time MODY9 diagnosis.

\section{Methods}

Clinical data and routine test results of this patient, admitted to the Department of Endocrinology in Beijing Children hospital, were collected, summarized, analyzed and discussed. In search for a monogenic cause of the early onset of the disease, gene sequencing was performed.

DNA extraction: $2 \mathrm{~mL}$ of peripheral venous blood was collected (EDTA anticoagulant), and genomic DNA was extracted using the QIAamp whole blood DNA extraction kit (Qiagen, Germany).

Genetic examination and technical route of exon gene detection: Whole-exon sequencing analysis after informed consent from the patient's parents was conducted. DNA was extracted from the peripheral blood of the subjects to construct the genome library. The biotin-labelled probe was hybridized with the library DNA under certain conditions, and the streptavidin-modified magnetic beads were covalently bound to the biotin-labelled probe to capture the target gene. Finally, magnetic beads carrying the target gene were adsorbed by magnetic frame, eluted, purified and enriched. The enriched target genes were sequenced by NextSeq 500 high-throughput sequencer (Illumina). The sequencing data were compared with the hG19 reference sequence of the human genome using BWA software, and the comparison files were sorted, filtered, and compared locally with multiple sequences to eliminate false positives. Sanger sequencing was used to verify gene variants associated with the clinical phenotype of the subject. Genetic analyses were completed by Beijing McGinow Technology Co., LTD.

\section{Results}

Clinical data: The proband was male, aged 19 months. He was admitted to the hospital on $20^{\text {th }}$ December 2018, due to excessive drinking, polyuria for overhalf a month and wheezing for 3 days. The medical history showed that the child was given birth by a G1P1 woman with a caesarean section in the $39^{\text {th }}$ gestational week. Birth weight and length were $3.35 \mathrm{~kg}$ and $51 \mathrm{~cm}$, respectively. The health status during the neonatal period as well as the child's mental and physical development was normal More than half a month before admission, the patient had polydipsia and polyuria without known inducement, and his daily water intake significantly increased compared to before. 2 days before admission, the child presented with a severe wheezing accompanied by Kussmaul respiration and a poor general condition. He was sent to the local hospital and was admitted to the intensive care unit (ICU) for treatment. After admission, his random blood glucose levels were $40 \mathrm{mmol} / \mathrm{L}$, urine ketone body was positive, and the preliminary given diagnosis was "diabetes, diabetic ketoacidosis". Due to an unsatisfactory therapeutic effect, the child was transferred to the Beijing Children hospital for further diagnosis and treatment. On arrival at the emergency unit, the intravenous blood glucose level was $20.74 \mathrm{mmol} / \mathrm{L}$, and blood gas analysis resulted in $\mathrm{pH} 7.07, \mathrm{PCO}_{2}$ $17.5 \mathrm{mmHg}, \mathrm{BE}-23.5 \mathrm{mmol} / \mathrm{L}$, afterwards the patient was admitted to the endocrinology department for further treatment. The patient had no family history, and the blood glucose levels of both parents were normal.

Physical examination on admission : Patient's temperature was 37, respiration rate was 37 times/min, pulse was 150 times/min, blood pressure was $116 / 62 \mathrm{mmHg}$. The patient's weight was $12 \mathrm{~kg}$ with a normal physical development. He was in a poor general condition, with decreased level of consciousness, no tears when crying, an anaemic appearance, Kussmaul respiration and a fruity breath odor. His lips and skin were dry with slightly low skin elasticity. The right upper lung presented with moist carackles. The upper 
extremities were cool to the elbows, while the lower extremities were cool to the knees, with a dilated blood vessel pattern on the limbs and a CRT of 3 seconds.

Laboratory and imaging results: The blood sugar was 21.69mmol/L (Normal range: $3.9-6.1 \mathrm{mmol} / \mathrm{L}$ ), glycosylated haemoglobin was $11.1 \%$ (Reference value: $4-6 \%$ ), insulin level was $0.56 \mu \mathrm{IU} / \mathrm{ml}$ at the $0^{\text {th }}$ $\min (6.0-27.0 \mu \mathrm{IU} / \mathrm{ml})$, C-peptide level was $0.09 \mathrm{ng} / \mathrm{ml}$ at minute $0(1.1-5.0 \mathrm{ng} / \mathrm{ml})$. Anti-islet cell (ICA) and anti-glutamate decarboxylase antibodies (GADA) were negative. Blood gas analysis was repeated: $\mathrm{pH}$ was 7.06 , the carbon dioxide partial pressure was $15 \mathrm{mmHg}$, the oxygen partial pressure was $74 \mathrm{mmHg}$, the actual and standard hydrogen carbonates were $4.2 \mathrm{mmol} / \mathrm{L}$ and $5.8 \mathrm{mmol} / \mathrm{L}$, respectively, the total carbon dioxide was $4.7 \mathrm{mmol} / \mathrm{L}$, the extracellular fluid base storage $26.1 \mathrm{mmol} / \mathrm{L}$, and whole blood base storage was $24.2 \mathrm{mmol} / \mathrm{L}$. Blood biochemistry showed that potassium was $3.24 \mathrm{mmol} / \mathrm{L}$, sodium was $136 \mathrm{mmol} / \mathrm{L}$, chlorine was $109.9 \mathrm{mmol} / \mathrm{L}$, while the osmotic pressure was $296 \mathrm{mOsm} / \mathrm{kg}$. The urea was $2.35 \mathrm{mmol} / \mathrm{L}$, creatinine was $30 \mathrm{umol} / \mathrm{L}$, total calcium was $2.53 \mathrm{mmol} / \mathrm{L}$, lipase was $9.2 \mathrm{u} / \mathrm{L}$, amylase was $50 \mathrm{U} / \mathrm{L}$ and D-3 hydroxybutyric acid was $10.18 \mathrm{mmol} / \mathrm{L}$. Concentrations of insulin-like growth factor were $<25.0 \mathrm{ng} / \mathrm{ml}$, while the insulin-like growth factor binding globulin was $0.56 \mathrm{~g} / \mathrm{ml}$. Urine routine analyses showed that the glucose was $4+$, ketone was $3+$, the protein was $1+$, while the urobilinogen and nitrite were negative, occult blood trace was negative and centrifugation microscopy showed no erythrocyte/HPF. The serum cortisol at $8 \mathrm{AM}$ was $>50.0 \mu \mathrm{g} / \mathrm{dl}$, and adrenocorticotrophic hormone at $8 \mathrm{AM}$ was $68.8 \mathrm{pg} / \mathrm{ml}$. Thyroid analysis showed that the total serum levels of T3 and T4 were $19.77 \mathrm{ng} / \mathrm{dl}$ and $2.04 \mathrm{ug} / \mathrm{dl}$, respectively. The serum thyroid-stimulating hormone had a blood concentration of $0.391 \mathrm{mIU} / \mathrm{L}$, serum-free T3 and T4 were $3.52 \mathrm{pmol} / \mathrm{L}$ and $5.51 \mathrm{pmol} / \mathrm{L}$. Chest $\mathrm{X}$-ray showed slightly more texture and fuzziness in the lungs with patchy shadow on the right upper lung. Abdominal ultrasound showed no abnormalities.

After admission, the patient was diagnosed with Type 1 diabetes, diabetes ketoacidosis and pneumonia. After fluid infusion, ketone correction, insulin and anti-infection treatment the patient gradually recovered.After 17 days of hospitalization, the blood glucose level was stable and the patient was discharged. The total amount of insulin dosage at discharge was $7.5 \mathrm{IU}$, equivalent to $0.625 \mathrm{IU} / \mathrm{kg} / \mathrm{d}$.

Gene sequencing results: The genetic sequencing results revealed that the patient carried a rare c. $487 \mathrm{C}>\mathrm{T}$ heterozygous mutation in the $7^{\text {th }}$ exon region of the PAX4 gene. This mutation led to a R163W variation in the amino acid sequence. The patient's father carried the same heterozygous mutation while the mother presented with a normal genotype, suggesting that the mutation could be autosomal dominant. The estimated frequency of the mutation in the normal population database was 0.00010 , which is a low-frequency mutation. There were no related reports of this mutation in the HGMD database. SIFT, PolyPhen_2, Mutation Taster, GERP ++ and REVEL respectively predicted this mutation as harmful, harmful, benign, harmful and benign. According to the ACMG guideline, the mutation was preliminarily determined to be of unknown clinical significance.

Gene sequencing results of the proband and his parents are shown in Figures 1, 2 and 3.

\section{Discussion}

MODY9 is a rare type of MODY caused by a PAX4 gene mutation. In vertebrates, the PAX family consists of nine members, which can be divided into four subgroups according to the specific composition of the structural domain. Among them, the subfamily IV (PAX4 and PAX6) is composed of paired and homologous domains [12]. PAX4 gene is located on 7q32.1 and contains 10 exons and 9 introns with a molecular weight of 7,851bp. Its encoding product is the paired box protein PAX-4. PAX-4 protein contains a paired domain $(\mathrm{PD})$ and a homeobox domain (HD), both of which are potential DNA-binding domains $\left(\mathrm{DBD}_{\mathrm{S}}\right)$ [13]. PAX4 protein is widely distributed, mainly expressed in islet pancreatic cells, and plays an important regulatory role in the production of islet progenitor cells and the differentiation of islet $\beta$-cells and islet $\delta$-cells $[11,14]$. It can inhibit the expression of $a$-cell genes and promote the connection of endocrine cells with $\beta$-cells and $\delta$ -cells. As a key regulator of mammalian pancreatic development, PAX4 protein first appeared in endocrine 
progenitor cells at 9.5 days of embryos and then is selectively expressed in $\beta$-cells [15]. In $\beta$-cells, PAX4 protein can maintain the expression of pancreatic and duodenal homeobox 1, Pdx1 and NK6 homeobox 1, Nkx 6.1, both of which are important regulators of pancreatic $\beta$-cell development [16].

In animal experiments, heterozygous PAX4 knockout mice had almost no mature $\beta$-cells and $\delta$-cells, and a large number of abnormal $\alpha$-cells were found. All of which could lead to an increase in blood glucose levels, suggesting that PAX4 is a key regulator in the differentiation process of precursor cells into different islet cell [17]. Other research data showed that PAX4 homogenous variation can cause the destruction of PAX4 protein's targeting ability, resulting in an reduced inhibitory function and an increase in $a$-cells, which promotes glucagon production and leads to increased blood sugar $[18,19]$.

In 2007, Nattachet Plengvidhya et al. studied the pathogenic genes of 46 MODY probands (all Thai origin) applying DNA direct sequencing techniques [10]. They reported a missense mutation of the PAX4 gene with a variation of R164W and a shear mutation of PAX4 gene responsible for a IVS7-1variation in two diabetic families, respectively, being the first two reports on PAX4 mutation in relation to MODY and was defined as MODY9 [10]. Nattachet Plengvidhya et al. found that 4 members of the diabetic family with the R164W variation carried the same mutation. Three of which suffered from diabetes. The onset age of the proband was 20, while in the other two cases it was 29 and 50. No ketosis, nor ketoacidosis were detected at the onset of the disease. Another family member developed impaired glucose tolerance at age 14, which did not progress to diabetes. Patients in this group were treated by diet control or oral hypoglycemic drugs, and their blood glucose level were well controlled. 6 members of the diabetic family with IVS7-1 variation presented with diabetes, of which only the proband underwent gene sequencing analysis and was found to carry the mutation. Of the 6 members with diabetes, 1 developed retinopathy and kidney disease 10 years after diagnosis of diabetes, and 3 died of end-stage renal failure at the age of 52-53 years [10].

MODY9 is rarely seen in clinical practice, and only a few families have been reported so far. In 2011, Wakako Jo et al reported a Japanese family with MODY9 [20]. There were 2 patients with DM in this family, all of whom carried a deletion mutation in the third exon region of PAX4 C374-412del39. The proband's disease onset was at the age of 15 with ketosis and had been treated with insulin since diagnosis. Another patient in the family, the father of the proband, was diagnosed at the age of 30 with mild diabetes, which was well controlled through diet management only. In 2018, Serena Pezzilli et al. reported a missense mutation of PAX4 c.593C > T (A198V) in a MODY9 Italian family with 4 diabetic patients. Among them, the proband and the sister carried Ala198Val missense mutation, while the proband's mother and aunt did not undergo gene sequencing. The 4 members were diagnosed as early as 20 years old and as late as 60 years old. Three of them were treated with oral medication and their blood glucose was well controlled, while the other one received insulin treatment [21].

Three more missense mutations of the PAX gene were found in subsequent studies. The missense mutation of c.377A > G (p. D126G) and c.55C > T (p.R19W) was reported by Natalia Zubkova et al. [22]. C.92G>T (p.r.31l) missense mutation was reported by Aaron Chapla et al. in India [23]. So far, the inheritance pattern of MODY9 mutation has been mainly autosomal dominant.

In this study, we report a disease that started in infancy and was characterized by an autosomal dominant mutation, which was consistent with the characteristics of MODY. C.487C $>$ T missense mutation was found in the $7^{\text {th }}$ exon region of the PAX4 gene in children, leading to a variation of p.R163W in the amino acid sequence. The R163W variation is located in the homologous domain of the PAX4 gene, which is responsible for the binding of PAX4 protein to the target DNA sequence and is also associated with conserved amino acid residues between species. R163W variation causes the replacement of polar amino acids by non-polar amino acids, which is suspected to impair the inhibitory effect of PAX4 protein on insulin and glucagon promoters [24], leading to hyperglycaemia and diabetes. This mutation has not been reported in previously published papers. Genetic analysis of the family members showed that the father of the patient carried the same heterozygous mutation, while the mother presented with a normal genotype, suggesting that the mutation was autosomal dominant, and the inheritance pattern was consistent with previous literature reports. 
The estimated frequency of $\mathrm{C} .487 \mathrm{C}>\mathrm{T}$ missense mutation in the PAX4 gene in the normal population database is 0.00010 , which is a low-frequency mutation. According to clinical manifestations and sequencing results, the patient was diagnosed with MODY9 monogenic diabetes. The father of the proband had no diabetes at that time, which may be related to epigenetic factors or incomplete genetic penetrance.

Previous literature showed that the onset age of MODY9 patients ranged from 14 to 50 years. The clinical manifestations of patients with the same mutation were also significantly different. Mild cases may only present with elevated fasting glucose levels or impaired glucose tolerance, while some cases manifest with typical symptoms of diabetes such as polydipsia, polyuria and emaciation, only few cases occur with ketoacidosis. In this study, the onset of illness occurred during early childhood, characterized by typical diabetic symptoms accompanied by ketoacidosis. In which case was a MODY9 with early onset and severe clinical symptoms. Islet autoantibody was negative, and fasting insulin and C-peptide levels were significantly reduced, which was consistent with the clinical characteristics of MODY9 reported in previous literature.

MODY9 is generally treated the same way as other types of MODY, mainly with diabetic diet, exercise and insulin therapy. Some patients are responsive to oral hypoglycemic agents. In some patients, treatment has to adjusted according to the progression of the disease. On the latest follow-up, the patient had been diagnosed with diabetes for 17 months. He was treated with basic and mealtime insulin injections and his blood sugar levels were in normal range ever since.

The clinical outcome of MODY9 patients varies greatly. In mild cases, the only symptom may be impaired glucose tolerance, without progression to diabetes, and in severe cases, renal or retinal complications may occur, or even death from end-stage renal failure.

In summary, in Chinese children, the heterozygous mutation of c.487C $>\mathrm{T}$ in the PAX4 gene can lead to the occurrence of MODY9. MODY9 patients differ in onset ages and clinical symptoms, making it difficult to distinguish them from other MODY subtypes. Here, we conclude that gene sequencing analysis is of great significance in the diagnosis and classification of MODY, and an accurate genetic diagnosis will enable patients to receive more specialized treatment.

\section{Author Contribution}

Di Zhang analysed the clinical data and wrote the manuscript. Wenli Yang and Yanmei Sang contributed to the review and revision of the manuscript. Li Ren and Yanmei Sang were involved in the treatment and follow-up of the patient.

\section{Conflict of Interest}

The authors declare no potential conflict of interest relevant to this article.

\section{Ethical Approval}

This research was approved by the Ethics Committee of Beijing Children's Hospital and written informed consent was obtained from the patient's parents.

\section{Funding Information}

There was no funding for this research.

\section{Acknowledgements}

We are grateful to the patient and his family for their participation in this study. We thank Beijing McGinow Technology Co. for their technical support in gene sequencing and data analysis. We would also like to acknowledge the clinical staff of the Endocrinology Department of Beijing Children's Hospital and the Neonatal Department of Capital Institute of Pediatrics for their assistance in the present study.

[1] Shepherd, M.et al.Systematic Population Screening, Using Biomarkers and Genetic Testing, Identifies 2.5\% of the U.K.Pediatric Diabetes Population With Monogenic Diabetes.Diabetes Care, 39: 1879-1888, doi:10.2337/dc16-0645 (2016). 
[2] Bansal, V.et al.Spectrum of mutations in monogenic diabetes genes identified from high-throughput DNA sequencing of 6888 individuals. BMC medicine 15, 213, doi:10.1186/s12916-017-0977-3 (2017).

[3] Anik A, et al. Maturity-onset diabetes of the young(MODY): an update[J].J Pediatr Endocrinol Metab, 2015, 28(34): 25l-263. DOI:10.1515/jpem-2014-0384.

[4] Froguel P,Vaxillaire M. Monogenic diabetes in the young, pharmacogenetics and relevance to multifactorial forms of type 2 diabetes.[J]. 2008, 29(3).

[5] Hattersley AT, SAW G, Polak M, et al. ISPAD clinical practice consensus guidelines 2018: the diagnosis and management of monogenic diabetes in children and adolescents[J]. Pediatr Diabetes, 2018,19 Suppl 27: 47-63. DOI: $10.1111 /$ pedi. 12772

[6] FlannickJ,Beer NL,Bick AG,et al.Assessing the phenotypic effects in the general population of rare variants in genes for a dominant Mendelian form of diabetes[J].Nat Genet, 2013, 45(11):1380-1385.

[7] Bowman P, Flanagan S, Edghill E, et al. Heterozygous ABCC8 mutations are a cause of MODY[J].Diabetologia, 2012,55(1): 123-127.

[8] Bonnefond A, Philippe J, Durand E, et al. Whole-exome sequencing and high through put genotyping identified KCNJ11 as the thirteenth MODY gene[J].PLoS One,2012,7(6):e37423.

[9] Fajans SS, Bell GI, Polonsky KS (2001) Molecular mechanisms and clinical pathophysiology of maturityonset diabetes of the young.N Engl J Med, 345(13):971-980

[10] Plengvidhya N, Kooptiwut S, Songtawee N, et al. PAX4 mutations in Thais with maturity onset diabetes of the young[J]. J ClinEndocrinol Metab, 2007, 92(7): 2821-2826.

[11] Brun T, Franklin I, St-Onge L, Biason-Lauber A, Schoenle EJ, Wollheim CB et al (2004) The diabeteslinked transcription factor PAX4 promotes beta-cell proliferation and survival in rat and human islets. J Cell Biol 167(6):1123-1135.

[12] Mansouri A, Goudreau G, Gruss P. Pax genes and their role in organogenesis. Cancer Res, 1999;59: $1707-9$.

[13] Antojenifer Panneerselvam, Arun Kannan, et al. PAX proteins and their role in pancreas. Diabetes research and clinical practice 155(2019)107792;

[14] Sosa-Pineda B, ChowdhuryK, Torres M, et al. The Pax4 gene is essential for differentiation of insulinproducing beta cells in the mammalian pancreas[J]. Nature,1997,386(6623):399-402. DOI: 10.1038/386399a0

[15] Habener JF, Kemp DM, Thomas MK. 2005 Minireview transcriptional regulation in pancreatic development. Endocrinology 146:1025-1034.

[16] Wang J, Elghazi L, Parker SE, Kizilocak H, Asano M, Sussel L, Sosa-Pineda B 2004 The concerted activities of Pax4 and Nkx2.2 are essential to initiate pancreatic $\beta$-cell differentiation. Dev Biol, 266:178 -189 .

[17] Sosa-Pineda B, Chowdhury K, Torres M, Oliver G, Gruss P 1997 The Pax4 gene is essential for differentiation of insulin-producing $\beta$ cells in the mam-malian pancreas. Nature 386:399-402

[18] T Zhang, HW Wang, et al. Pax4 synergistically acts with Pdx1, Ngn3 and MafA to induce HuMSCs to differentiate into functional pancreatic $\beta$-cells. Experimental and Therapeutic Medicine. 2019, 18: 25922598 .

[19] Marc Gregory Yu, Hillary A. Keenan, Hetal S. Shah, et al. Residual $\beta$ cell function and monogenic variants in long-duration type 1 diabetes patients. The Journal of Clinical Investigation. 2019;129(8):32523263. 
[20] Jo W, Endo M, Ishizu K, et a1. A novel PAX4 mutation in a Japanese patient with maturity-onset diabetes of the young[J]. Tohoku J Exp Med, 2011, 223(2): 113-118.

[21] Serena Pezzilli, Ornella Ludovico(2018), Insights From Molecular Characterization of Adult Patients of Families With Multigenerational Diabetes. Diabetes 2018;67:137-145

[22] Natalia Zubkova, Fatima Burumkulova2, et al. High frequency of pathogenic and rare sequence variants in diabetes-related genes among Russian patients with diabetes in pregnancy, Acta Diabetologica 2019,https://doi.org/10.1007/s00592-018-01282-6

[23] Aaron Chapla, Mahesh Doddabelavangala Mruthyunjaya, et al. Maturity onset diabetes of the young in India - a distinctive mutation pattern identified through targeted next-generation sequencing, Clinical Endocrinology (2014), DOI: 10.1111/cen.12541

[24] Smith SB, Ee HC, Conners JR, German MS 1999 Paired-homeodomain transcription factor PAX4 acts as a transcriptional repressor in early pancreatic development. Mol Cell Biol, 19:8272-8280.

\section{Figure Legends}

Fig.1 PAX4 gene sequencing of the patient. The $7^{\text {th }}$ exon region carries a heterozygous mutation of c.487C $>t$, leading to a variation of $\mathrm{R} 163 \mathrm{~W}$ in the amino acid sequence.

Fig.2 PAX4 gene sequencing of the patient's father. The 7th exon region carries a heterozygous mutation of $\mathrm{c} .487 \mathrm{C}>\mathrm{t}$, leading to a variation of $\mathrm{R} 163 \mathrm{~W}$ in the amino acid sequence.

Fig.3 PAX4 gene sequencing of the patient's mother. She presented with a normal genotype and did not carry the c.487C $>$ t heterozygous mutation.
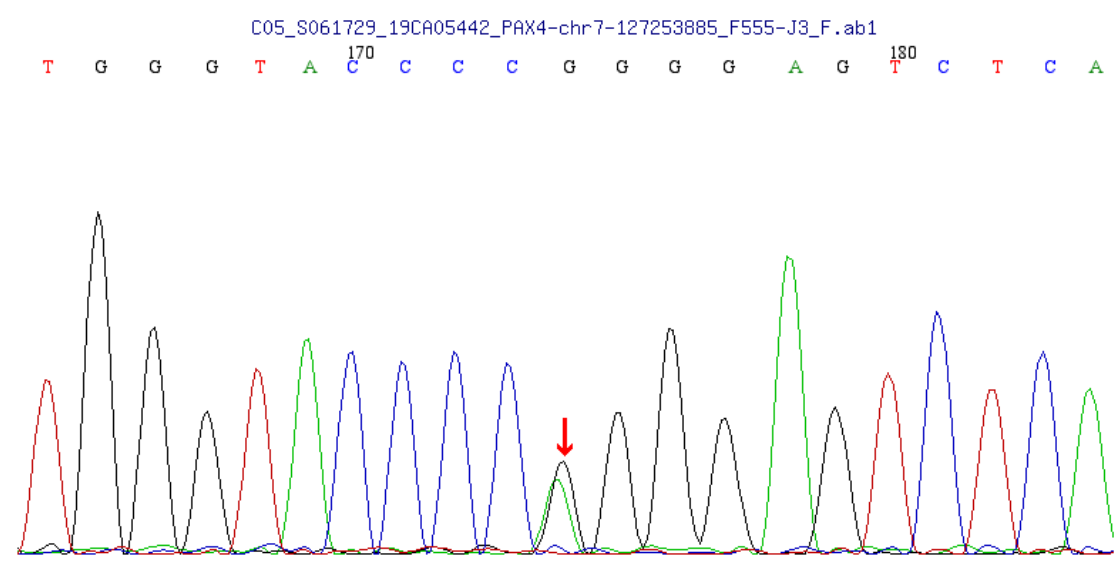

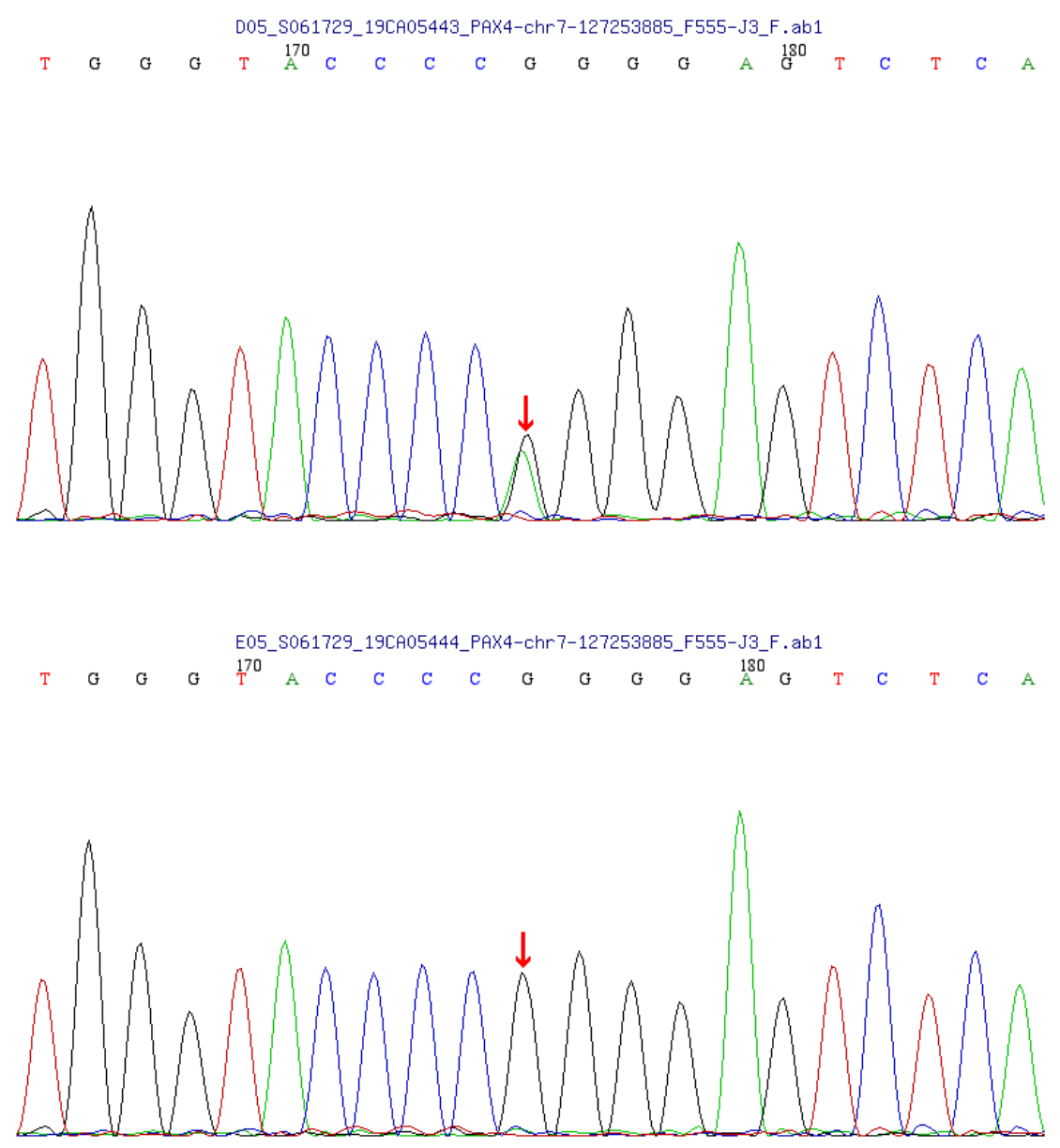